UCI-TR-2008-26

\title{
Thermal Relics in Hidden Sectors
}

\author{
Jonathan L. Feng, Huitzu Tu, and Hai-Bo Yu \\ Department of Physics and Astronomy, \\ University of California, Irvine, CA 92697, USA
}

\begin{abstract}
Dark matter may be hidden, with no standard model gauge interactions. At the same time, in WIMPless models with hidden matter masses proportional to hidden gauge couplings squared, the hidden dark matter's thermal relic density may naturally be in the right range, preserving the key quantitative virtue of WIMPs. We consider this possibility in detail. We first determine modelindependent constraints on hidden sectors from Big Bang nucleosynthesis and the cosmic microwave background. Contrary to conventional wisdom, large hidden sectors are easily accommodated. A flavour-free version of the standard model is allowed if the hidden sector is just $30 \%$ colder than the observable sector after reheating. Alternatively, if the hidden sector contains a 1-generation version of the standard model with characteristic mass scale below $1 \mathrm{MeV}$, even identical reheating temperatures are allowed. We then analyze hidden sector freezeout in detail for a concrete model, solving the Boltzmann equation numerically and understanding the results from both observable and hidden sector points of view. We find that WIMPless dark matter indeed obtains the correct relic density for masses in the range $\mathrm{keV} \lesssim m_{X} \lesssim \mathrm{TeV}$. The upper bound results from the requirement of perturbativity, and the lower bound assumes that the observable and hidden sectors reheat to the same temperature and is raised to the $\mathrm{MeV}$ scale if the hidden sector is 10 times colder. WIMPless dark matter therefore generalizes the WIMP paradigm to the largest mass range possible for viable thermal relics and provides a unified framework for exploring dark matter signals across nine orders of magnitude in dark matter mass.
\end{abstract}

PACS numbers: 95.35.+d, 04.65.+e, 12.60.Jv 


\section{INTRODUCTION}

At present, all solid evidence for dark matter is gravitational. At the same time, the possibility that dark matter has electromagnetic or strong interactions is highly constrained [1, 2]. A straightforward possibility, then, is that dark matter is hidden, consisting of particles that have no standard model (SM) gauge interactions.

Hidden sectors have a long and distinguished history. For example, the idea that a hidden sector may restore parity on cosmological scales is as old as the idea of parity violation itself [3]. Such hidden sectors, containing "mirror matter," have been studied by many groups, as have their possible implications for dark matter [4, 5, 6, 7, 8, 9, 10, 11, 12, 13]. String theory also motivates hidden sectors with their own gauge interactions [14], as in the case of the heterotic string [15] and intersecting brane models [16]. More recently, hidden sectors have been central to several phenomenological developments, including Higgs portals [17, 18], hidden valleys [19], unparticles [20], and quirks [21], and the possibility that dark matter may reside in a hidden sector has been discussed in several recent works [22, 23, 24, 25, 26, 27, 28, 29, 30, 31].

In considering hidden dark matter, one would seemingly be sacrificing critical virtues of more conventional dark matter candidates, namely, the connection of dark matter to the gauge hierarchy problem, and the fact that weakly-interacting massive particles (WIMPs) naturally have the desired thermal relic density. This is not necessarily true, however. In the

recently proposed framework of WIMPless dark matter [30], hidden sector dark matter also naturally has the desired thermal relic density. The essential idea is that, very generally, a dark matter candidate that decoupled from the thermal bath non-relativistically has a thermal relic density

$$
\Omega h^{2} \sim \frac{1}{\left\langle\sigma_{A} v\right\rangle} \sim \frac{m^{2}}{g^{4}}
$$

where $\left\langle\sigma_{A} v\right\rangle$ is the thermally-averaged annihilation cross section times velocity, and $g$ and $m$ are the dark matter particle's gauge coupling and mass. For WIMPs with weak interaction coupling constant $g_{\text {weak }} \simeq 0.65$ and weak-scale mass $m_{\text {weak }} \sim 100 \mathrm{GeV}-1 \mathrm{TeV}$, this relic mass density is naturally near the observed $\Omega_{\mathrm{DM}} h^{2} \simeq 0.11$.

In WIMPless models, hidden sector particles $X$ have masses and couplings that may be very different from WIMPs, but which nevertheless satisfy

$$
\frac{m_{X}}{g_{X}^{2}} \sim \frac{m_{\text {weak }}}{g_{\text {weak }}^{2}} .
$$

In the WIMPless examples discussed in Ref. [30], Eq. (2) follows from the structure of gauge-mediated supersymmetry breaking (GMSB), which generates hidden sector masses that are proportional to couplings squared. The WIMPless framework therefore naturally generalizes the WIMP paradigm to other cold dark matter candidates without sacrificing the key thermal relic density virtue of WIMPs. If additional connector particles with both SM and hidden gauge quantum numbers are present, they will mediate SM-hidden interactions. The WIMPless framework may therefore also predict qualitatively new signals, such as an explanation of the DAMA signal in terms of GeV dark matter [32].

In this paper, we study hidden thermal relics in general, and WIMPless dark matter in particular. For the most part, we assume that there are no connector particles, so that the hidden sector interacts with the SM only very weakly. In Sec. III, we carry out a modelindependent analysis of constraints on hidden sectors from Big Bang nucleosynthesis (BBN), 
the cosmic microwave background (CMB), and other cosmological data. As is well known, these exclude the possibility that the hidden sector is an exact copy of the SM [33]. This statement assumes that the hidden and observable sectors are at the same temperature at late times, however. In the present context, there is no strong reason to assume that the observable and hidden sectors are reheated to the same temperature [5, 6], and, even if they are, for a truly decoupled hidden sector, their temperatures will differ by the time of BBN. Following previous works in the context of mirror matter (see, e.g., Refs. [5, 6, 8, 10, 34]), we consider the cosmological constraints allowing different temperatures and find that even large hidden sectors are easily accommodated. For example, a hidden version of the SM is allowed even if the hidden sector's reheating temperature is as much as $70 \%$ of the observable sector's. Alternatively, if the hidden sector contains a 1-generation version of the MSSM with characteristic mass scale below $1 \mathrm{MeV}$, even identical reheating temperatures are allowed.

We then present a concrete model for the hidden sector in Sec. III and study the freezeout of dark matter in this hidden sector in Sec. IV] The relations of Eqs. (11) and (2) are, of course, only rough relations capturing the parametric dependences. In these Sections, we present a detailed analysis of a specific case that highlights several subtleties and generalizes previous freezeout analyses to cases with sectors at different temperatures. This analysis confirms the validity of conclusions based on Eqs. (11) and (2) and highlights various subtleties of the WIMPless framework. In particular, we find that WIMPless dark matter may have the desired thermal relic density for the entire range of $\mathrm{keV} \lesssim m_{X} \lesssim \mathrm{TeV}$, where the lower bound is set by the requirement that dark matter freezeout is non-relativistic, and the upper bound follows from the requirement of perturbative gauge couplings. WIMPless dark matter therefore encompasses as large a range of masses as one could expect of dark matter that has the naturally correct thermal relic density, and it provides a unified framework for addressing many diverse dark matter signals and phenomenology.

Finally, in Sec. V, we discuss modifications from the presence of connector particles with both hidden and observable sector gauge interactions. We find that, under general assumptions, these fields do not upset the conclusions derived earlier. We present our conclusions in Sec. VI.

\section{BBN AND CMB CONSTRAINTS}

We assume that the observable sector is the minimal supersymmetric standard model (MSSM), which is supplemented by a single hidden sector. Hidden sector parameters are denoted by the superscript $h$. For most of this work, we assume that there are no connectors, fields with both SM and hidden gauge charges. The observable and hidden sectors therefore interact extremely weakly with each other, and the observable sector's temperature $T$ need not equal the hidden sector's temperature $T^{h}$. We define

$$
\xi(t)=\frac{T^{h}(t)}{T(t)}
$$

to parametrize the mismatch. In addition, it will be convenient to introduce the standard relativistic degrees of freedom parameters. Letting

$$
C_{i}= \begin{cases}1, & i=\text { boson } \\ \frac{7}{8}, & i=\text { fermion }\end{cases}
$$


we define

$$
g_{*}(T) \equiv \sum_{m_{i}<T} C_{i} g_{i}\left(\frac{T_{i}}{T}\right)^{4} \quad g_{* S}(T) \equiv \sum_{m_{i}<T} C_{i} g_{i}\left(\frac{T_{i}}{T}\right)^{3},
$$

where the sum is over observable sector particles, and

$$
g_{*}^{h}\left(T^{h}\right) \equiv \sum_{m_{i}<T^{h}} C_{i} g_{i}\left(\frac{T_{i}}{T^{h}}\right)^{4} \quad g_{* S}^{h}\left(T^{h}\right) \equiv \sum_{m_{i}<T^{h}} C_{i} g_{i}\left(\frac{T_{i}}{T^{h}}\right)^{3}
$$

where the sum is over hidden sector particles. $T_{i}$ is the temperature of particle $i$, and $g_{i}$ denotes its internal degrees of freedom. Throughout this work, we assume that the gravitino has a negligible relic density, as is the case, for example, for low and moderate reheating temperatures, and the gravitino is not included Eqs. (5) and (6).

$\mathrm{BBN}$ is sensitive to the expansion rate of the Universe at time $t_{\mathrm{BBN}} \sim 1$ s and temperature $T_{\mathrm{BBN}} \sim 1 \mathrm{MeV}$. The expansion rate is determined by the energy density, and so constrains light particles even if they have no SM interactions [35, 36]. The constraint is conventionally quoted as a bound on $N_{\text {eff }}$, the effective number of light neutrino species, and may be taken to be $N_{\text {eff }}=3.24 \pm 1.2(95 \%$ CL), where the baryon density has been fixed to the value determined by the $\mathrm{CMB}$, and both ${ }^{4} \mathrm{He}$ and $\mathrm{D}$ data are included [37, 38]. In our framework, this implies

$$
g_{*}^{h}\left(T_{\mathrm{BBN}}^{h}\right)\left(\frac{T_{\mathrm{BBN}}^{h}}{T_{\mathrm{BBN}}}\right)^{4}=\frac{7}{8} \cdot 2 \cdot\left(N_{\mathrm{eff}}-3\right) \leq 2.52(95 \% \mathrm{CL}),
$$

where $T_{\mathrm{BBN}}^{h}$ is the temperature of the hidden sector at time $t_{\mathrm{BBN}}$. If the hidden sector is an exact copy of the MSSM and $T_{\mathrm{BBN}}^{h}=T_{\mathrm{BBN}}, g_{*}^{h}\left(T_{\mathrm{BBN}}^{h}\right)=10.75$. This violates the bound of Eq. (7), as is well-known [33], and may create the impression that large hidden sectors of the size of the MSSM are completely excluded.

If the observable and hidden sectors are not in thermal contact, however, the hidden sector may be colder than the observable sector. This would be the case if, for example, the inflaton couplings to the observable and hidden sectors are not identical, so that they reheat to different temperatures [5, 6]. Alternatively, the observable and hidden sectors may initially have the same temperature, either because they have the same inflaton couplings or because they are in thermal contact, but may cool independently and have different temperatures at later times. In fact, it quite reasonable that the observable and hidden sectors lose thermal contact before BBN. Thermal contact would require efficient interactions between the two sectors at low temperatures. These would require light connector fields to mediate these interactions, but light fields with SM gauge charges generically violate experimental constraints. We conclude, then, that it is far from guaranteed that the observable and hidden sectors will be at the same temperature at $t_{\mathrm{BBN}}$; generically, they will be at different temperatures.

To explore this possibility, we assume that the observable and hidden sectors reheat at time $t_{\mathrm{RH}}$ to temperatures $T_{\mathrm{RH}}$ and $T_{\mathrm{RH}}^{h}$, respectively. We further assume that the entropy per comoving volume is conserved in each sector separately, so that

$$
\frac{g_{* S}^{h}\left(T_{\mathrm{BBN}}^{h}\right) T_{\mathrm{BBN}}^{h 3}}{g_{* S}^{h}\left(T_{\mathrm{RH}}^{h}\right) T_{\mathrm{RH}}^{h 3}}=\frac{g_{* S}\left(T_{\mathrm{BBN}}\right) T_{\mathrm{BBN}}^{3}}{g_{* S}\left(T_{\mathrm{RH}}\right) T_{\mathrm{RH}}^{3}} .
$$

Given mass spectra in the observable and hidden sectors, reheat temperatures $T_{\mathrm{RH}}$ and $T_{\mathrm{RH}}^{h}$, and Eq. (8) , the ratio $T_{\mathrm{BBN}}^{h} / T_{\mathrm{BBN}}$ is fixed, and one can determine if Eq. (7) is satisfied. 
To provide numerical examples, we define

$$
\xi_{\mathrm{RH}} \equiv \frac{T_{\mathrm{RH}}^{h}}{T_{\mathrm{RH}}},
$$

the ratio of temperatures just after reheating, and assume $T_{\mathrm{RH}}$ is above the mass of all MSSM particles. We also define

$$
g_{\text {light }} \equiv \sum_{m_{i}<T_{\mathrm{BBN}}} C_{i} g_{i} \quad g_{\text {heavy }} \equiv \sum_{T_{\mathrm{BBN}}<m_{i}<T_{\mathrm{RH}}} C_{i} g_{i}
$$

for the observable sector and

$$
g_{\text {light }}^{h \mathrm{BBN}} \equiv \sum_{m_{i}<T_{\mathrm{BBN}}^{h}} C_{i} g_{i} \quad g_{\text {heavy }}^{h \mathrm{BBN}} \equiv \sum_{T_{\mathrm{BBN}}^{h}<m_{i}<T_{\mathrm{RH}}^{h}} C_{i} g_{i}
$$

for the hidden sector, where we have assumed all hidden relativistic degrees of freedom have a common temperature. With these definitions, Eq. (8) becomes

$$
\frac{g_{\text {light }}^{h \mathrm{BBN}}}{g_{\text {light }}^{h \mathrm{BBN}}+g_{\text {heavy }}^{h \mathrm{BBN}}}\left(\frac{T_{\mathrm{BBN}}^{h}}{T_{\mathrm{BBN}}}\right)^{3}=\frac{g_{\text {light }}}{g_{\text {light }}+g_{\text {heavy }}} \xi_{\mathrm{RH}}^{3} .
$$

Solving for $T_{\mathrm{BBN}}^{h} / T_{\mathrm{BBN}}$ and substituting the MSSM values $g_{\text {light }}=10.75$ and $g_{\text {light }}+g_{\text {heavy }}=$ 228.75, we find that the BBN bound of Eq. (7) becomes

$$
g_{\text {heavy }}^{h \mathrm{BBN}} \leq\left(\frac{3.49}{\xi_{\mathrm{RH}}}\right)^{3}\left(g_{\text {light }}^{h \mathrm{BBN}}\right)^{\frac{1}{4}}-g_{\text {light }}^{h \mathrm{BBN}} .
$$

Bounds in the $\left(g_{\text {light }}^{h \mathrm{BBN}}, g_{\text {heavy }}^{h \mathrm{BBN}}\right)$ plane for various values of $\xi_{\mathrm{RH}}$ are given in Fig. 1. Several aspects deserve comment. As evident from Eq. (13), the bound is extremely sensitive to $\xi_{\mathrm{RH}}$. The $\xi_{\mathrm{RH}}=0.5$ contour is barely contained on the plot, implying that if the hidden sector reheats to half the temperature of the observable sector, very large hidden sectors are allowed. Several example hidden sectors, defined precisely in Sec. III, are also given on the plot. Model A is a 1-generation flavor-free version of the MSSM, with all Yukawa couplings of order 1 . We see that it is allowed for $\xi_{\mathrm{RH}}<0.92$, that is, reheat temperatures that are almost identical to the observable sector. This is our prototype model, which will be used in our detailed freezeout analysis in Sec. IV Model B is a full 3-generation flavor-free version of the MSSM. We see that even a hidden sector as "large" as the MSSM is allowed, provided $\xi_{\mathrm{RH}}<0.74$.

Perhaps even more interesting, Fig. 1 shows that Model C, a 1-generation version of the MSSM with all degrees of freedom relativistic at BBN, is allowed, even for $\xi_{\mathrm{RH}}=1.06$. Naively, one might expect a hidden sector that starts at the same temperature as the MSSM and has over 100 light degrees of freedom to be completely excluded. In fact, however, such a hidden sector is much colder than the MSSM at BBN times, since its cooling is not slowed by the disappearance of heavy degrees of freedom, in contrast to the MSSM. This cooling is critical, given the 4th power of the temperature in Eq. (7), and makes such a hidden sector allowed. For similar reasons, Fig. 1 shows that an excluded hidden sector is only made more excluded by the addition of heavy degrees of freedom. Note, however, that Fig. 1 also shows that in some cases, rather counter-intuitively, an excluded hidden sector may be made allowed by the addition of light degrees of freedom. 


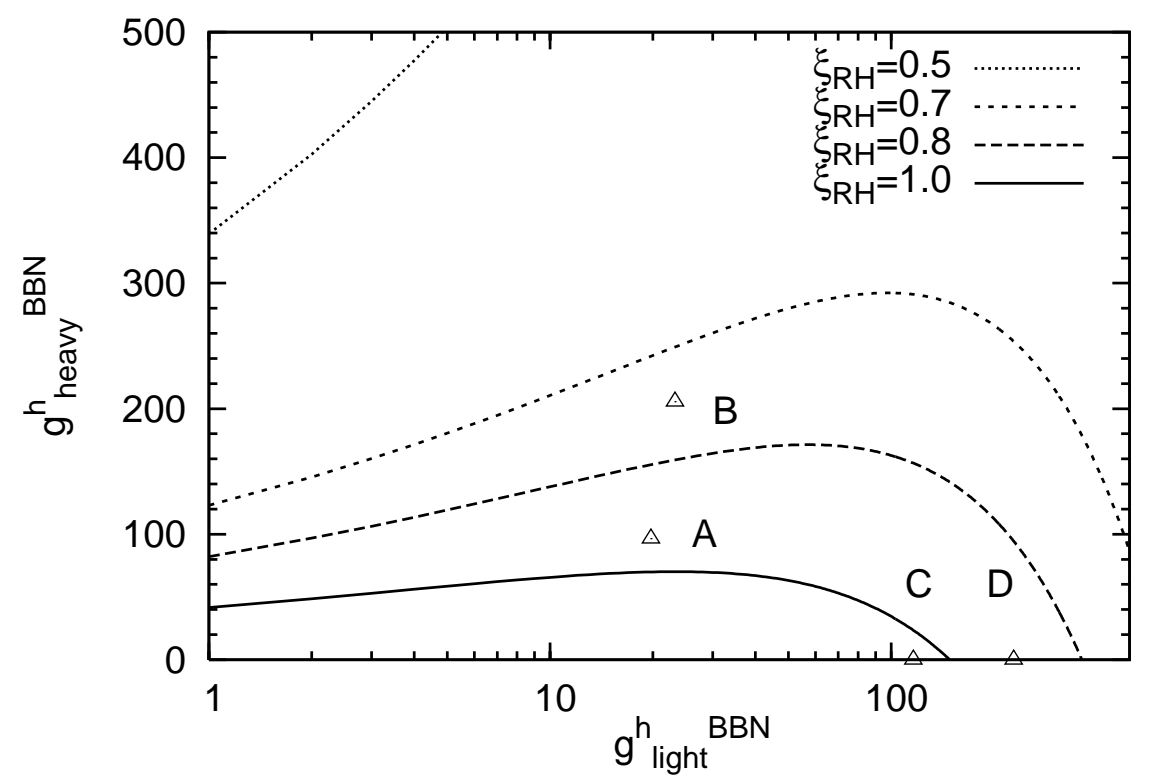

FIG. 1: Bounds from BBN in the $\left(g_{\text {light }}^{h \mathrm{BBN}}, g_{\text {heavy }}^{h \mathrm{BBN}}\right)$ plane, where $g_{\text {light }}^{h \mathrm{BBN}}$ and $g_{\text {heavy }}^{h \mathrm{BBN}}$ are the hidden degrees of freedom with masses $m<T_{\mathrm{BBN}}^{h}$ and $T_{\mathrm{BBN}}^{h}<m<T_{\mathrm{RH}}^{h}$, respectively, for $\xi_{\mathrm{RH}} \equiv T_{\mathrm{RH}}^{h} / T_{\mathrm{RH}}=0.5,0.7,0.8,1.0$ (from top to bottom). The regions above the contours are excluded. We assume that the observable sector reheats to a temperature above the mass of all MSSM particles. The values of $\left(g_{\text {light }}^{h \mathrm{BBN}}, g_{\text {heavy }}^{h \mathrm{BBN}}\right)$ are marked for four example hidden sectors: (A) 1-generation and (B) 3-generation flavor-free versions of the MSSM with $T_{\mathrm{BBN}}^{h}<m_{X}<T_{\mathrm{RH}}^{h}$, and (C) 1-generation and (D) 3-generation flavor-free versions of the MSSM with $m_{X}<T_{\mathrm{BBN}}^{h} / 2$ (see Sec. III).

CMB temperature anisotropy measurements provide another constraint on the number of relativistic species. WMAP 5 year data, combined with distance information from baryon acoustic oscillations, supernovae (SNIa) and Hubble constant measurements, imply $N_{\text {eff }}=$ $4.4 \pm 1.5$ (68\% CL) [39]. In contrast to the BBN bound, this is a $68 \%$ CL bound; at 95\% CL, the bound is very weak. This bound is therefore numerically much weaker than the BBN bound. In the future, however, CMB data from, for example, the Planck satellite [40] will achieve a much higher sensitivity in constraining the amount of radiation energy [41, 42, 43, 44. At the same time, of course, the CMB bound constrains the energy density at much later times with redshift $z \sim 1000-3000$ and observable sector temperatures $T \sim 1 \mathrm{eV}$. It is thus interesting to explore the CMB constraint when the hidden sector includes particles with mass between $1 \mathrm{MeV}$ and $1 \mathrm{eV}$. (See also Ref. [45] for a combined constraint.)

The current CMB constraint in our framework is

$$
g_{*}^{h}\left(T_{\mathrm{CMB}}^{h}\right)\left(\frac{T_{\mathrm{CMB}}^{h}}{T_{\mathrm{CMB}}}\right)^{4}=\frac{7}{8} \cdot 2 \cdot\left(N_{\mathrm{eff}}-3.046\right)\left(\frac{T_{\nu}}{T_{\gamma}}\right)^{4} \leq 1.30(68 \% \mathrm{CL}),
$$

where $T_{\nu} / T_{\gamma}=(4 / 11)^{1 / 3}$ is the neutrino to photon temperature ratio after the era of $e^{ \pm}$ annihilation. The fact that the SM neutrinos were not fully decoupled at $e^{ \pm}$annihilation is taken into account by using the modified number of SM neutrinos of 3.046 instead of 


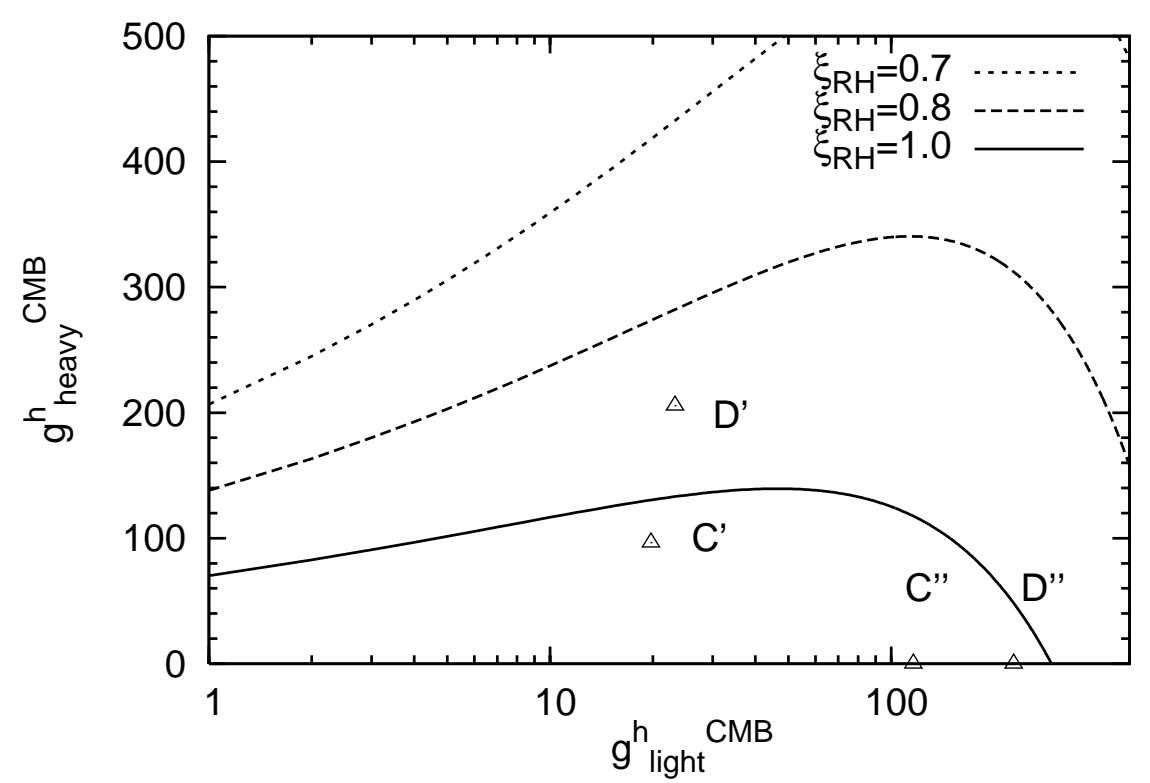

FIG. 2: As in Fig. 1, but for bounds from the CMB in the $\left(g_{\text {light }}^{h \mathrm{CMB}}, g_{\text {heavy }}^{h \mathrm{CMB}}\right)$ plane, where $g_{\text {light }}^{h \mathrm{CMB}}$ and $g_{\text {heavy }}^{h \mathrm{CMB}}$ are the hidden effective degrees of freedom with masses $m<T_{\mathrm{CMB}}^{h}$ and $T_{\mathrm{CMB}}^{h}<m<T_{\mathrm{RH}}^{h}$, respectively. The values of $\left(g_{\text {light }}^{h \mathrm{CMB}}, g_{\text {heavy }}^{h \mathrm{CMB}}\right)$ are given for four example hidden sectors: $\left(\mathrm{C}^{\prime}\right) 1$ generation and $\left(\mathrm{D}^{\prime}\right)$ 3-generation flavor-free versions of the MSSM with $T_{\mathrm{CMB}}^{h}<m_{X}<T_{\mathrm{RH}}^{h}$, and $\left(\mathrm{C}^{\prime \prime}\right)$ 1-generation and $\left(\mathrm{D}^{\prime \prime}\right)$ 3-generation flavor-free versions of the MSSM with $m_{X}<T_{\mathrm{CMB}}^{h} / 2$ (see Sec. III).

3 [46, 47]. Following the BBN analysis, this CMB bound implies

$$
g_{\text {heavy }}^{h \mathrm{CMB}} \leq\left(\frac{4.14}{\xi_{\mathrm{RH}}}\right)^{3}\left(g_{\text {light }}^{h \mathrm{CMB}}\right)^{\frac{1}{4}}-g_{\text {light }}^{h \mathrm{CMB}},
$$

where $g_{\text {heavy }}^{h \mathrm{CMB}}$ and $g_{\text {light }}^{h \mathrm{CMB}}$ are defined as in Eq. (11), but with $T_{\mathrm{BBN}}^{h}$ replaced by $T_{\mathrm{CMB}}^{h}$.

We show current CMB bounds in the $\left(g_{\text {light }}^{h \mathrm{CMB}}, g_{\text {heavy }}^{h \mathrm{CMB}}\right)$ plane in Fig. 2, along with four example models. Figure 1 s Model $\mathrm{C}$, with all degrees of freedom light compared to $T_{\mathrm{BBN}}^{h}$, is now differentiated into many models, depending on how many of the degrees of freedom are light compared to $T_{\mathrm{CMB}}^{h}$. These define a line in Fig. 2, with endpoints given by Models $\mathrm{C}^{\prime}$ and $\mathrm{C}^{\prime \prime}$. We find that if these models are allowed by the BBN constraints, they are also allowed by the current CMB constraint. Similarly, Fig. 1 $\mathrm{s}$ Model D is differentiated into Models $\mathrm{D}^{\prime}$ and $\mathrm{D}^{\prime \prime}$ in Fig. 2, but again we find that in such models, if the BBN constraint is satisfied, the current CMB constraint is also.

In these models, then, the current $\mathrm{CMB}$ constraint is never as stringent as the BBN constraint. This may change with the strengthening of the CMB constraint in the future. In addition, it is interesting that in the presence of hidden sectors, there may be many particles with mass between $T_{\mathrm{BBN}}^{h}$ and $T_{\mathrm{CMB}}^{h}$, and $\mathrm{BBN}$ and the CMB yield independent information about the hidden sector mass spectrum. This contrasts with the case of the SM, where CMB constraints may be viewed as consistency checks on BBN results. 


\section{A CONCRETE MODEL}

In Sec. II, we found that hidden sectors may contain many degrees of freedom without violating cosmological bounds. With this as motivation, in the following section, we will examine freezeout in hidden sectors whose temperatures may differ from the observable sector's. To prepare the way, in this section we first specify a model and mass spectrum for the hidden sector.

The WIMPless models of Ref. [30] are supersymmetric, with supersymmetry breaking mediated by gauge interactions. A worthwhile endeavor would be to build a complete GMSB WIMPless model. In this study, however, we instead work with a "GMSB-inspired" model. We do this for two reasons. First, our primary aim here is to clarify cosmological issues. Building a complete GMSB model with a WIMPless hidden sector, complete with $\mu$ term solution and other particle physics details, would take us too far afield, especially since, as we will see, the relic density is independent of many of these details. Second, as noted in Sec. I, the essential virtue of WIMPless models, that they have the naturally correct thermal relic density, relies solely on the fact that masses in the hidden sector are proportional to gauge couplings squared. This is true for GMSB, of course, but is also valid in many other contexts, such as anomaly-mediated supersymmetry breaking models, ${ }^{1}$ lessening the motivation for detailed consideration of any one GMSB model.

The model we consider has an MSSM-like hidden sector, with $\mathrm{SU}(3) \times \mathrm{SU}(2) \times \mathrm{U}(1)$ gauge interactions. In contrast to the MSSM, however, we assume that all Yukawa couplings are $\mathcal{O}(1)$. We refer to this as a flavor-free version of the MSSM. For simplicity, we will focus on a 1-generation flavor-free version of the MSSM, but, as all Yukawa couplings are large, we will use 3rd generation nomenclature for this one generation of hidden matter fermions and sfermions.

All hidden superpartners are assumed to have mass $\sim m_{X}$, the superpartner mass scale. In addition, since $m_{X}$ also sets the scale for the Higgs boson parameters, the hidden Higgs bosons $h^{h}, H^{h}, A^{h}$, and $H^{h \pm}$ also have this mass, as do the hidden weak gauge bosons $W^{h}$ and $Z^{h}$. Given $\mathcal{O}(1)$ Yukawa couplings, the hidden fermions $\tau^{h}$, $b^{h}$, and $t^{h}$ are therefore also at the superpartner mass scale. The light, effectively massless particles are the hidden photon $\gamma^{h}$, gluon $g^{h}$ and neutrino $\nu^{h}$. As is typical of GMSB, the gravitino is also light, and its relic density from late decays to the gravitino is negligible. We further assume low or moderate reheating temperatures so that the abundance of gravitinos produced during reheating is also insignificant.

WIMPless dark matter requires that one particle at the $m_{X}$ mass scale be stable. We assume that the lightest $m_{X}$ scale particle is the hidden right-handed stau $X \equiv \tilde{\tau}_{R}^{h}$. This is the lightest superpartner in much of GMSB parameter space, and it is also reasonable to assume that it is lighter than the $\tau^{h}$, given $\mathcal{O}(1)$ Yukawa couplings, just as the stop may be lighter than the top in the MSSM. The stau's stability is then guaranteed by hidden sector electric charge conservation. One might worry about the viability of a charged dark matter candidate. Efficient Compton scattering of hidden staus on hidden photons $\tilde{\tau}_{R}^{h \pm} \gamma^{h} \rightarrow \tilde{\tau}_{R}^{h \pm} \gamma^{h}$ would lead to dissipative energy loss, making the dark matter particles behave more like baryons. However, this scattering cross section is $\sigma \sim g^{h 4} / m_{X}^{2}$, which, given Eq. (2), is as weak as the SM weak interactions. Hidden sector charged dark matter is therefore consistent with the known properties of dark matter from structure formation.

\footnotetext{
${ }^{1}$ We thank Raman Sundrum for making this point.
} 
Given the few light states, there are only a few dark matter annihilation channels:

$$
\tilde{\tau}_{R}^{h+} \tilde{\tau}_{R}^{h-} \rightarrow \nu^{h} \bar{\nu}^{h}, \gamma^{h} \gamma^{h}, \gamma^{h} Z^{h} .
$$

The neutrino channel is mediated by an $s$-channel $Z^{h}$. The gauge boson final states are produced through processes with $\tilde{\tau}_{R}^{h}$ in $t$ - and $u$-channels and 4-point $\tilde{\tau} \tilde{\tau} \gamma \gamma$ and $\tilde{\tau} \tilde{\tau} \gamma Z$ vertices. The relic density is therefore dependent on only a few of the many supersymmetric parameters, which we will now discuss. Note that we assume no left-right stau mixing.

All three gauge couplings enter the analysis. The $\mathrm{SU}(2)$ and $\mathrm{U}(1)$ gauge couplings enter the annihilation cross sections. The SU(3) gauge coupling must also be specified, because it determines the number of relativistic degrees of freedom at BBN. For simplicity, for the hidden gauge couplings, we adopt assumptions motivated by unification. Gauge coupling unification implies that the hidden weak mixing angle is $\tan \theta_{W}^{h}=\sqrt{3 / 5}$ at the GUT scale. In principle, this is modified by renormalization group ( $R G)$ evolution. The RG equations for the hidden sector MSSM gauge couplings are

$$
\frac{d g_{a}^{h}}{d \ln (Q / M)}=\frac{1}{16 \pi^{2}} b_{a}^{h} g_{a}^{h 3},
$$

where $b_{a}^{h}=(13 / 5,-3,-7)$ for the 1 -generation case, and $b_{a}^{h}=(33 / 5,1,-3)$ for the 3 generation case. As we will see in Sec. IV] however, WIMPless models are valid in the ranges $\mathrm{keV} \lesssim m_{X}<\mathrm{TeV}$ and $10^{-5} \lesssim g_{X}\left(m_{X}\right)<1$, depending on the reheating temperature ratio $\xi_{\mathrm{RH}}$. For most of this range, then, $g_{X}\left(m_{X}\right) \lesssim 0.1$, and the fractional change in the gauge couplings from RG evolution is $\left|\Delta g_{a}^{h} / g_{a}^{h}\right| \approx\left|b_{a}^{h}\right|\left(g_{a}^{h 2} / 16 \pi^{2}\right) \ln \left(M_{\text {mess }} / m_{X}\right) \lesssim 0.01$; that is, the gauge couplings evolve very little. Given this, we will assume $\tan \theta_{W}^{h}=\sqrt{3 / 5}$ at $m_{X}$. For $m_{X} \gtrsim 100 \mathrm{GeV}$, this is not the prediction of unification, and our assumption therefore implicitly assumes some more complicated structure.

The unification assumption also typically implies that the hidden confinement scale

$$
\Lambda_{\mathrm{QCD}}^{h} \sim m_{X} e^{\frac{8 \pi^{2}}{b_{3}^{h} g_{3}^{h 2}\left(m_{X}\right)}}
$$

is far below $T_{\mathrm{BBN}}^{h}$. For example, for the 1-generation case, taking $m_{X} \sim 100 \mathrm{GeV}$ and $g_{3}\left(m_{X}\right)=0.5$, we find $\Lambda_{\mathrm{QCD}}^{h} \sim 10^{-9} \mathrm{eV}$. We therefore assume $\Lambda_{\mathrm{QCD}}^{h}<T_{\mathrm{BBN}}^{h}$, and so the hidden gluons contribute to $g_{*}^{h}$ at BBN. Again, this does not follow from unification for the largest $m_{X}$.

We will identify $g_{X}$ with the hidden SU(2) gauge coupling $g^{h}$. As for the hidden MSSM mass spectrum, we assume that all massive hidden particles have masses in the range $m_{X} \leq$ $m_{i} \leq 2 m_{X}$, and that all relativistic degrees of freedom are always at the same temperature. The effective relativistic degrees of freedom in the hidden sector are then

$$
g_{*}^{h}\left(T^{h}\right)=g_{* S}^{h}\left(T^{h}\right)= \begin{cases}116.25(228.75), & T^{h} \geq 2 m_{X} \\ 19.75(23.25), & T^{h} \leq m_{X}\end{cases}
$$

for the 1-generation (3-generation) case, and we linearly interpolate between these two values for $T^{h}$ between $m_{X}$ and $2 m_{X}$. The exact spectrum in this range is largely irrelevant for the relic abundance calculation, since we consider only dark matter that is non-relativistic at freezeout. Given these assumptions, the thermal relic density is completely determined by only four parameters:

$$
m_{X}, g_{X}, m_{Z}, \xi_{\mathrm{RH}} \equiv \frac{T_{\mathrm{RH}}^{h}}{T_{\mathrm{RH}}} .
$$




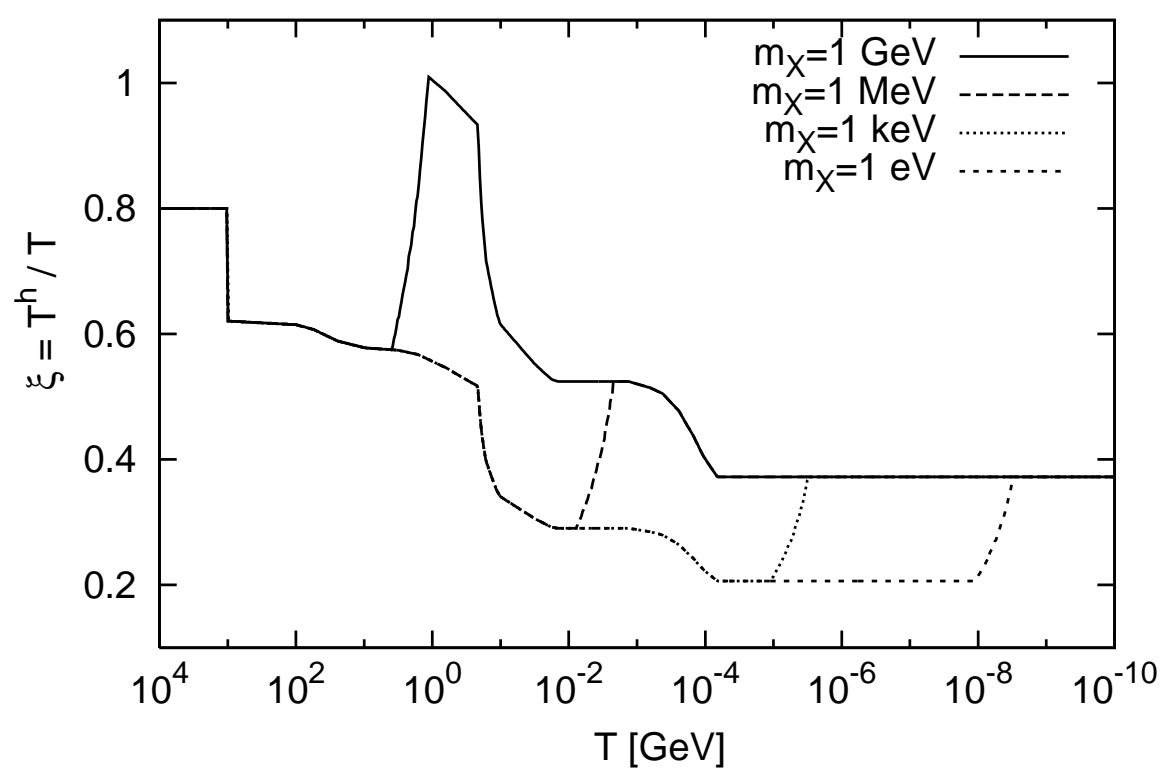

FIG. 3: Evolution of $\xi=T^{h} / T$, the ratio of hidden to observable temperatures, for $m_{X}=1 \mathrm{GeV}$, $1 \mathrm{MeV}, 1 \mathrm{keV}$ and $1 \mathrm{eV}$, assuming $T_{\mathrm{RH}}=50 \mathrm{TeV}, \xi_{\mathrm{RH}}=0.8$, and a hidden sector that is the 1-generation flavor-free version of the MSSM. For other $\xi_{\mathrm{RH}}$, these curves are simply re-scaled by $\xi_{\mathrm{RH}} / 0.8$. All supersymmetric particles in the observable sector are assumed to have mass around $1 \mathrm{TeV}$.

In Fig. 3 we plot the relative evolution of the observable and hidden photon temperatures for the cases $m_{X}=1 \mathrm{GeV}, 1 \mathrm{MeV}, 1 \mathrm{keV}$ and $1 \mathrm{eV}$. To make this plot, we must determine $T^{h}$ as a function of $T$ for a given $m_{X}$. To get the hidden sector temperature $T^{h}$ at a given observable sector temperature $T$, the value of $g_{* S}^{h}\left(T^{h}\right)$ is required, which is in fact unattainable unless one knows $T^{h}$. Our approach is the following: we first solve for $g_{* S}^{h}\left(T^{h}\right) T^{h 3}$ from Eq. (8) with $T_{\mathrm{BBN}}$ and $T_{\mathrm{BBN}}^{h}$ replaced by $T$ and $T^{h}$, respectively. We then determine $T^{h}$ by dividing this quantity by $g_{* S}^{h}\left(T^{h}\right)$ as determined by Eq. (19) in the three trial intervals $T^{h} \leq m_{X}, m_{X}<T^{h}<2 m_{X}$, and $2 m_{X}<T^{h}$, and choose the value of $T^{h}$ that yields a self-consistent solution.

The general feature of Fig. 3 is that as $T$ decreases from $T_{\mathrm{RH}}, \xi=T^{h} / T$ drops when observable degrees of freedom become non-relativistic and rises when hidden degrees of freedom become non-relativistic. Rapid drops occur at $T \sim \mathrm{TeV}$ when the observable superpartners become non-relativistic and $\xi \propto \sqrt[3]{g_{* S}(T)}$, and at $T \sim 250 \mathrm{MeV}$, where the QCD transition occurs. The rise occurs when the hidden $m_{X}$ mass particles become nonrelativistic at $T \sim 4 m_{X}-10 m_{X}$. At $T \sim m_{X}-5 m_{X}$, where the exact value depends on $m_{X}$, the hidden sector phase transition concludes, and $\xi$ begins to track $\sqrt[3]{g_{* S}(T)}$ again.

\section{THERMAL RELIC DENSITIES}

We are now ready to determine thermal relic densities in the case of observable and hidden sectors with unequal temperatures. For the hidden sector, we assume the 1-generation flavorfree version of the MSSM described in Sec. III. The temperature of the hidden sector thermal 
bath at any instant can be read off from Fig. 3 .

The number density of dark matter particles $n$ is determined by the competition between the expansion rate of the universe and the dark matter annihilation rate through the Boltzmann equation

$$
\frac{d n}{d t}=-3 H n-\left\langle\sigma_{A} v\right\rangle\left(T^{h}\right)\left[n^{2}-n_{\mathrm{eq}}^{2}\left(T^{h}\right)\right] .
$$

The Hubble parameter depends on the effective relativistic degrees of freedom in both the observable and hidden sectors and is

$$
H(T)=\left[\frac{4 \pi^{3} G_{N}}{45} g_{*}^{\text {tot }}(T) T^{4}\right]^{\frac{1}{2}},
$$

where

$$
g_{*}^{\text {tot }}(T)=g_{*}(T)+g_{*}^{h}\left(T^{h}\right)\left(\frac{T^{h}}{T}\right)^{4} .
$$

In contrast to $H,\left\langle\sigma_{A} v\right\rangle$, the thermally-averaged product of the total annihilation cross section and the Møller velocity, and $n_{\text {eq }}$, the equilibrium number density, are determined by the hidden temperature alone.

To solve the Boltzmann equation, it is standard to change to dimensionless variables through

$$
t \rightarrow x \equiv \frac{m_{X}}{T} \quad n \rightarrow Y \equiv \frac{n}{s} .
$$

These new variables are natural from the observable sector viewpoint, as they use the observable sector's temperature $T$ as the "clock," and the observable sector's entropy density $s=\frac{2 \pi^{2}}{45} g_{* S} T^{3}$ as the fiducial quantity characterizing the universe's expansion. Note, however, that in the present context there is also another useful change of variables,

$$
t \rightarrow x^{h} \equiv \frac{m_{X}}{T^{h}} \quad n \rightarrow Y^{h} \equiv \frac{n}{s^{h}},
$$

that is far more natural from the hidden sector point of view. We will return to this below.

In terms of the observable sector variables of Eq. (24), the Boltzmann equation becomes

$$
\frac{d Y}{d x}=-\lambda\left[Y^{2}-Y_{\mathrm{eq}}^{2}\left(x^{h}\right)\right]
$$

with the expansion and annihilation effects encapsulated in the quantity

$$
\lambda=\sqrt{\frac{\pi}{45 G_{N}}} \frac{g_{* S}(T)}{\sqrt{g_{*}^{\text {tot }}(T)}}\left(1+\frac{1}{3} \frac{T}{g_{* S}(T)} \frac{d g_{* S}(T)}{d T}\right) \frac{m_{X}}{x^{2}}\left\langle\sigma_{A} v\right\rangle\left(T^{h}\right) .
$$

We will solve this equation in the non-relativistic regime, where $x^{h} \gtrsim 3$. The equilibrium value of the number of dark matter particles in a comoving volume is

$$
Y_{\mathrm{eq}}\left(x^{h}\right) \equiv \frac{n_{\mathrm{eq}}\left(T^{h}\right)}{s(T)}=\frac{1}{s(T)} \frac{g}{2 \pi^{2}} \int_{m_{X}}^{\infty} \frac{\sqrt{E^{2}-m_{X}^{2}}}{e^{E / T^{h}}-1} E d E \approx \frac{45 x^{h 2}}{4 \pi^{4} g_{* S}(T)} g K_{2}\left(x^{h}\right) \xi^{3},
$$


where $g$ denotes the number of internal degrees of freedom of the dark matter particle, and the last approximation of Eq. (28) is valid for $x^{h} \gtrsim 3$. Following Ref. [50], the thermallyaveraged cross section times velocity is determined using

$$
\left\langle\sigma_{A} v\right\rangle\left(T^{h}\right)=\frac{1}{8 m_{X}^{4} T^{h} K_{2}^{2}\left(\frac{m_{X}}{T^{h}}\right)} \int_{4 m_{X}^{2}}^{\infty} d s \sigma_{A}(s) \sqrt{s}\left(s-4 m_{X}^{2}\right) K_{1}\left(\frac{\sqrt{s}}{T^{h}}\right) .
$$

In Eqs. (28) and (29), $K_{i}$ are modified Bessel functions of order $i$. Equation (29) was derived for particles in thermal equilibrium obeying Maxwell-Boltzmann statistics, but it is applicable to other statistics, provided $T^{h} \lesssim m_{X} / 3$.

We determine the $\tilde{\tau}_{R}^{h}$ total annihilation cross section $\sigma_{A}(s)$ with the help of the CalcHEP package [48, 49]. Expanding around $\epsilon \equiv\left(s-4 m_{X}^{2}\right) / 4 m_{X}^{2}$ (see Ref. [50]), we find

$$
\left\langle\sigma_{A} v\right\rangle\left(T^{h}\right)=\frac{g_{X}^{4}}{m_{X}^{2}}\left[a_{0}+a_{1}\left(\frac{m_{X}}{T^{h}}\right)^{-1}+a_{2}\left(\frac{m_{X}}{T^{h}}\right)^{-2}+\ldots\right],
$$

where

$$
\begin{aligned}
& a_{0}=\left[\frac{1}{8 \pi}+\frac{1}{4 \pi}\left(1-\frac{m_{Z^{h}}^{2}}{4 m_{X}^{2}}\right) \tan ^{2} \theta_{W}^{h}\right] \sin ^{4} \theta_{W}^{h} \\
& a_{1}=\frac{3}{2}\left[-\frac{1}{6 \pi}-\frac{1}{3 \pi} \tan ^{2} \theta_{W}^{h}+\frac{1}{12 \pi} \frac{1}{\cos ^{4} \theta_{W}^{h}\left[\left(-4+\frac{m_{Z h}^{2}}{m_{X}^{2}}\right)^{2}+\frac{m_{Z h}^{2} \Gamma_{Z^{h}}^{2}}{m_{X}^{4}}\right]}\right] \sin ^{4} \theta_{W}^{h},
\end{aligned}
$$

and $\Gamma_{Z^{h}}$ is the $Z^{h}$ boson decay width. In keeping with our non-relativistic approximation, we neglect the $a_{2}$ and higher order terms. For $m_{Z^{h}} \approx 2 m_{X}, \tilde{\tau}_{R}^{h+} \tilde{\tau}_{R}^{h-} \rightarrow \nu^{h} \bar{\nu}^{h}$ is greatly enhanced by the $s$-channel resonance. Off this resonance, however, the neutrino channel is typically sub-dominant, because it is $P$-wave suppressed, as can be seen above. In our numerical study, we fix $m_{Z^{h}}=1.5 m_{X}$. The $Z^{h}$ boson remains in equilibrium with the hidden thermal bath through the decay and inverse decay processes $Z^{h} \leftrightarrow \nu^{h} \bar{\nu}^{h}$.

We now present several results for $Y(x)$. Because of the "stiffness" of the Boltzmann equation of Eq. (26), we adopt the implicit trapezoidal method with adaptive step size, developed by the authors of the DarkSUSY package [51], to solve for $Y(x)$ numerically. We first examine the $\xi_{\mathrm{RH}}$ dependence of the thermal relic density. In Fig. 4, we plot $Y(x)$ for various values of $\xi_{\mathrm{RH}}, m_{X}=1 \mathrm{MeV}$, and $g_{X}=5.57 \cdot 10^{-4}$. The latter two parameters are naturally accommodated in the WIMPless GMSB framework and yield $\Omega_{X} h^{2}$ in the required range, as we will see below. As $\xi_{\mathrm{RH}}$ decreases, we see two effects. First, for a given $x$, the hidden sector is colder, and so $Y_{\text {eq }}(x)$ is smaller. Second, since freezeout occurs when $H \sim \Gamma=n\left\langle\sigma_{A} v\right\rangle$, when $\xi_{\mathrm{RH}}$ decreases and $n$ drops, freezeout occurs earlier. As can be seen from Fig. 4, these effects act against each other, and the thermal relic density is fairly insensitive to $\xi_{\mathrm{RH}}$.

These results may be understood more precisely using standard analytic approximation formulas for thermal relic freezeout. The equilibrium number density is

$$
Y_{\mathrm{eq}}=0.145\left(g / g_{* S}\right) x^{3 / 2} \xi^{3 / 2} e^{-x / \xi},
$$




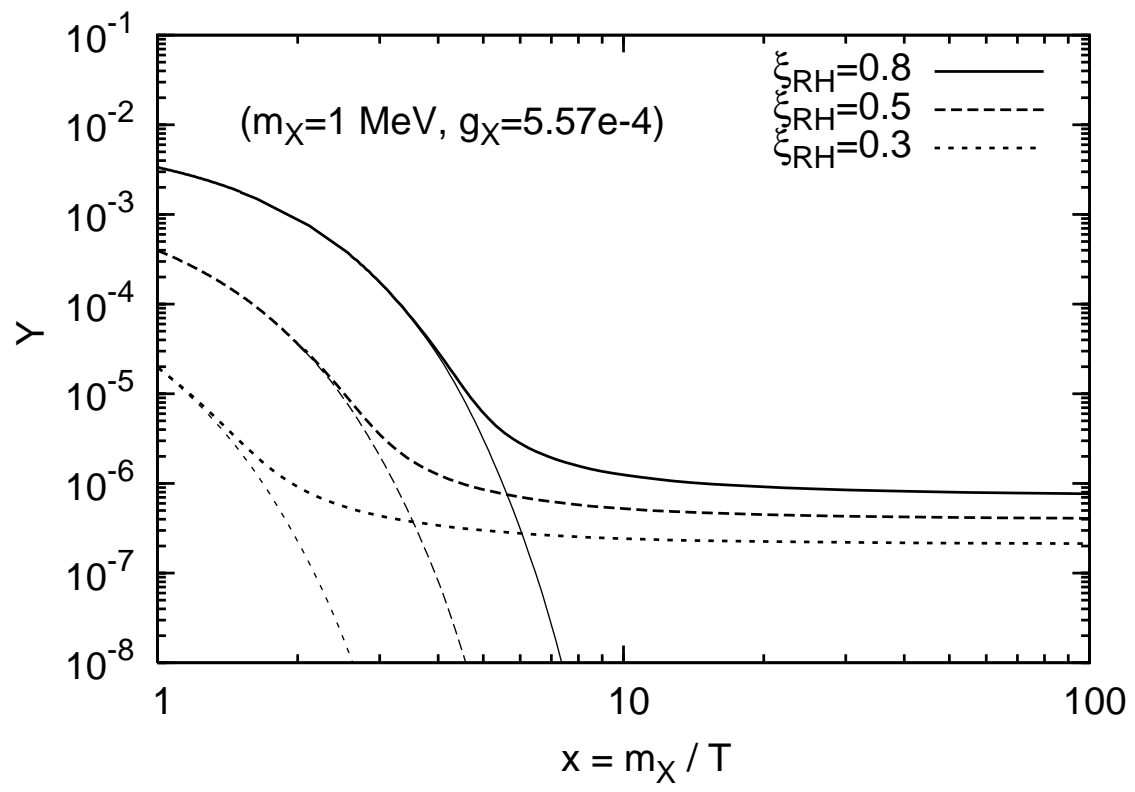

FIG. 4: $Y(x)$, the $\tilde{\tau}_{R}^{h}$ number density per comoving volume, as a function of $x \equiv m_{X} / T$, for $m_{X}=1 \mathrm{MeV}, g_{X}=5.57 \cdot 10^{-4}$, and various ratios of hidden sector to visible sector reheating temperature $\xi_{\mathrm{RH}}=0.8,0.5$ and 0.3 (from top to bottom).

where $\xi=T_{h} / T$, as defined above. Following the procedure given in Ref. [50] and assuming $S$-wave annihilation, an approximate expression for $x$ at freezeout is

$$
\begin{aligned}
x_{f} \approx & \xi \ln \left[0.038 M_{\mathrm{Pl}} m_{X} \sigma_{0}\left(g / \sqrt{g_{*}^{\text {tot }}}\right) \xi^{3 / 2} \delta(\delta+2)\right] \\
& -\frac{1}{2} \xi \ln \left\{\xi \ln \left[0.038 M_{\mathrm{Pl}} m_{X} \sigma_{0}\left(g / \sqrt{g_{*}^{\text {tot }}}\right) \xi^{3 / 2} \delta(\delta+2)\right]\right\},
\end{aligned}
$$

where $\sigma_{0}=a_{0} g_{X}^{4} / m_{X}^{2}$ and the parameter $\delta$ is tuned to make these analytical results fit the numerical results. ${ }^{2}$

For $S$-wave annihilation, the final relic abundance is approximately

$$
Y_{0} \approx \frac{3.79 x_{f}}{\left(g_{* S} / \sqrt{g_{*}^{\text {tot }}}\right) M_{\mathrm{Pl}} m_{X} \sigma_{0}}
$$

which depends linearly on $\xi$ through $x_{f}$. This linear dependence is verified by the numerical results presented in Fig. 4. The relative values of $Y_{0}$ obtained from Eq. (35) are $3.6: 1.9: 1$ for $\xi_{\mathrm{RH}}=0.8,0.5,0.3$, respectively, which almost exactly match the results from our numerical computation, which yields $3.5: 1.9: 1$.

The dependence on $\xi_{\mathrm{RH}}$ may also be understood from the hidden sector viewpoint. In Fig. 5, we consider the same model parameters as plotted in Fig. 4, but we now present $Y^{h}\left(x^{h}\right)$. From a hidden observer's point of view, the dependence on $\xi_{\mathrm{RH}}$ arises solely because $\xi_{\mathrm{RH}}$ determines how hot the observable sector is, and this impacts the expansion rate $H$. For

\footnotetext{
${ }^{2}$ For $\xi_{\mathrm{RH}}=0.3(0.8)$, we find that choosing $\delta=0.2(0.5)$ yields agreement typically better than $3 \%$. For larger $m_{X}$ or higher $\xi_{\mathrm{RH}}$, the final relic density is less sensitive to $\delta$.
} 


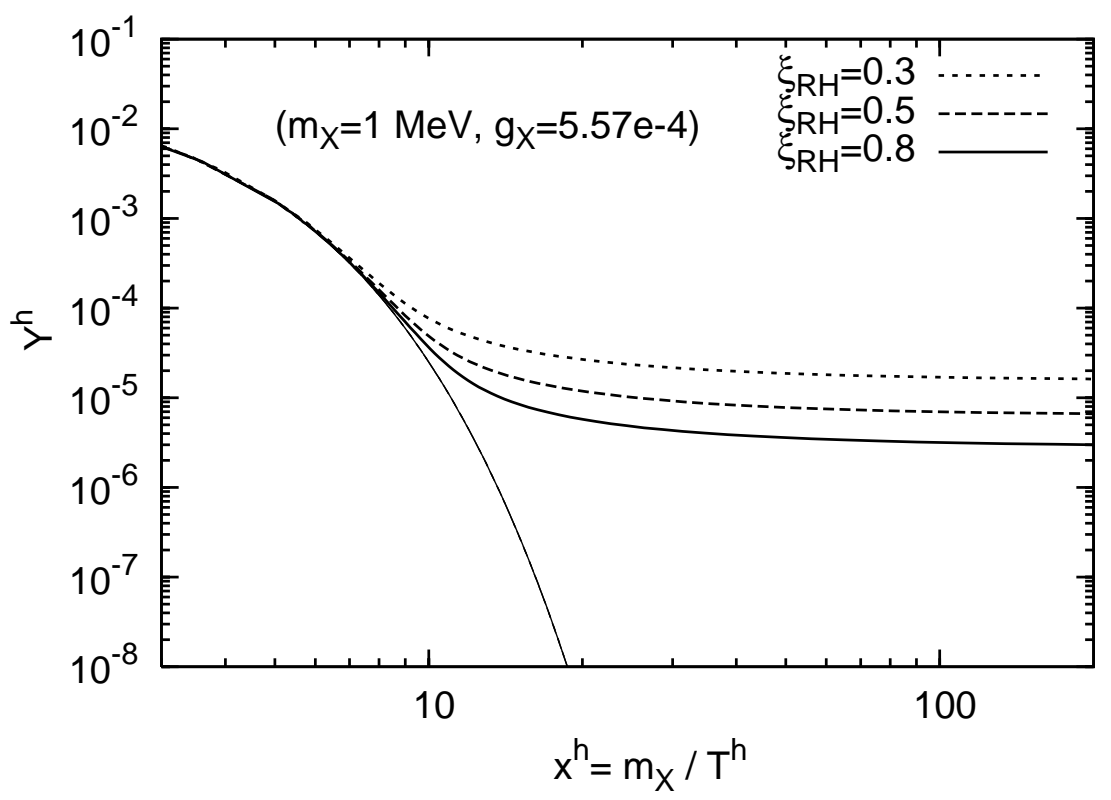

FIG. 5: As in Fig. 4, but now plotted in terms of the hidden sector parameters $Y^{h}\left(x^{h}\right)$.

a fixed $x^{h}$, lower $\xi_{\mathrm{RH}}$ implies higher $T$, faster expansion, and earlier freezeout. This is seen in Fig. 5. At first sight, the result that lower $\xi_{\mathrm{RH}}$ implies larger $Y^{h}$ may seem to contradict the results obtained earlier, where lower $\xi_{\mathrm{RH}}$ implies lower relic density. Note, however, that to convert $Y^{h}$ to a relic density, one must multiply $Y^{h}$ by the current $s^{h}$, which, unlike $s$, is not independent of $\xi_{\mathrm{RH}}$. For lower $\xi_{\mathrm{RH}}$, the current hidden sector temperature is lower, and the current $s^{h}$ is also lower. This effect makes the relic density smaller for lower $\xi_{\mathrm{RH}}$, in accord with our previous results derived from the observable sector viewpoint.

We now examine the dependence of the thermal freezeout process on $m_{X}$ and $g_{X}$. In Fig. 6, we plot $Y(x)$ for fixed $\xi_{\mathrm{RH}}$, but for a wide range of $\left(m_{X}, g_{X}\right)$ with $m_{X} \propto g_{X}^{2}$, the scaling relation of WIMPless scenarios. For low $m_{X}$, freezeout occurs earlier, as expected given Eq. (34). In our analysis, we have assumed that freezeout occurs in the non-relativistic regime, where $x^{h} \gtrsim 3$. Converting $x^{h}$ to $x$ using Fig. 3, we see from Fig. 6 that our analysis is self-consistent even for $m_{X}$ as low as $10 \mathrm{keV}$.

In Fig. 7 we present contours of constant $\Omega_{X} h^{2}$ in the $\left(m_{X}, g_{X}\right)$ plane. These contours result from our numerical analysis; although we have confined our previous plots to $x \lesssim 100$, the results of Fig. 7 use the relic density as determined at $x \sim 1000$, where it has truly stabilized.

Figure 7 summarizes all of our work so far, and there are several noteworthy features. First, we see that these contours essentially follow the scaling relation $m_{X} \propto g_{X}^{2}$. If, for example, $m_{X}$ is lowered by a factor of 4 and $g_{X}$ is lowered by a factor of 2 , the weakened interaction implies earlier freezeout and larger $Y_{0}$, but this compensates the lower $m_{X}$ to keep the relic density $\Omega_{X} h^{2} \propto m_{X} Y_{0}$ approximately invariant. Alternatively, using the analytic results presented above, this scaling relation keeps $\sigma_{A}$ invariant, and, given Eq. (35), this also keeps $\Omega_{X} h^{2}$ invariant. Of course, this is as expected from the general arguments given in Sec. I, but Fig. 7 shows that these arguments, based essentially on dimensional analysis, are in fact supported by a detailed calculation in the context of a concrete model with dark matter masses varying greatly and far beyond the conventional WIMP range. 


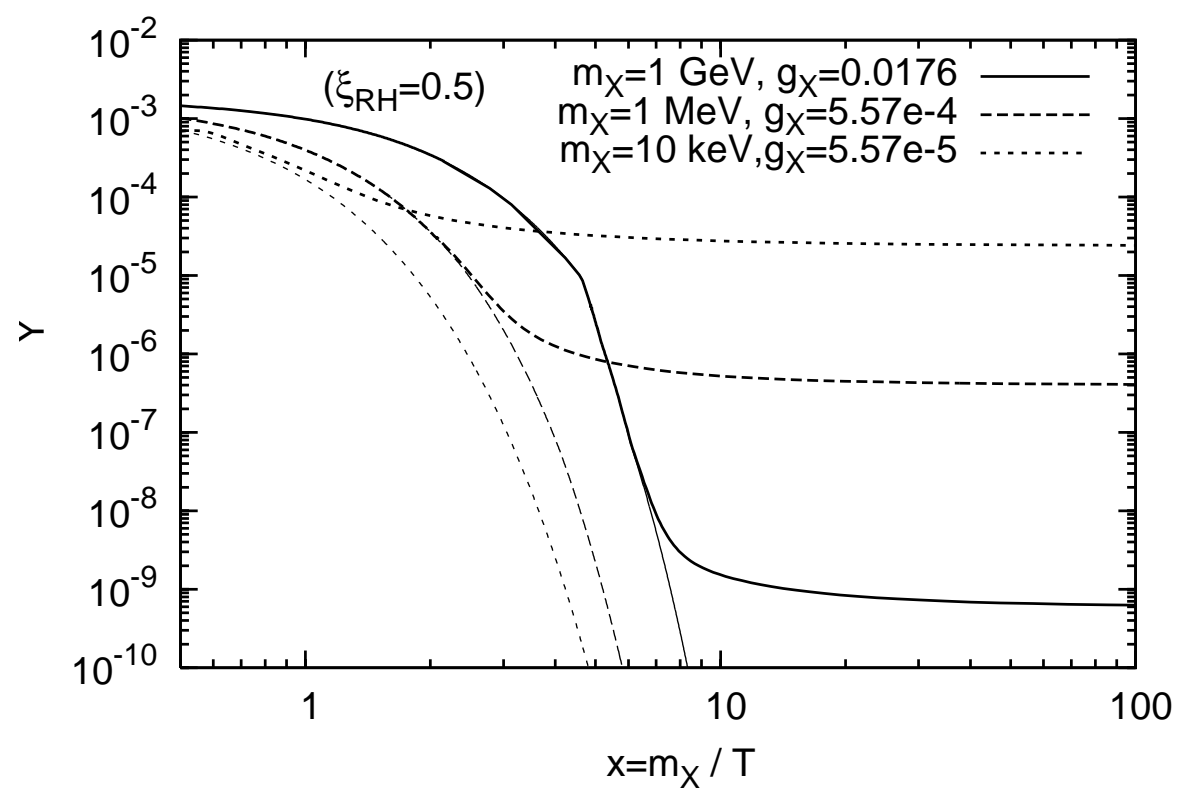

FIG. 6: $Y(x)$, the $\tilde{\tau}_{R}^{h}$ number density per comoving volume, as a function of $x \equiv m_{X} / T$, for fixed $\xi_{\mathrm{RH}}=0.5$ and $\left(m_{X}, g_{X}\right)=\left(1 \mathrm{GeV}, 1.76 \cdot 10^{-2}\right),\left(1 \mathrm{MeV}, 5.57 \cdot 10^{-4}\right)$, and $\left(10 \mathrm{keV}, 5.57 \cdot 10^{-5}\right)$. All cases are chosen to have the same ratio $m_{X} / g_{X}^{2}$.

Second, for comparison, Fig. 7 also includes two dashed contours to indicate the corresponding observable sector weak mass scale $m_{\text {weak }}$. The definition of $m_{\text {weak }}$ depends on how one chooses to characterize the natural weak scale in supersymmetric theories (the $\mu$ parameter, the Bino mass, etc.), and these depend on several parameters, such as the number of messengers and the messenger scale. We simply choose $m_{\text {weak }} \equiv\left(m_{X} / g_{X}^{2}\right) g^{\prime 2}$, where $g^{\prime}$ is the $\mathrm{U}(1)$ gauge coupling of the MSSM, and plot contours for $m_{\text {weak }}=100 \mathrm{GeV}$ and $1 \mathrm{TeV}$. The fact that there exist values in the $\left(m_{X}, g_{X}\right)$ plane that give the correct $\Omega_{X} h^{2}$ is, of course, not surprising. However, the fact that the two solid curves lie between the two dashed curves means that the preferred values of $\left(m_{X}, g_{X}\right)$ are those that correspond to models in which the observable sector's superpartners are at the weak scale.

We also see from Fig. 7 that, for a fixed $m_{X}$, lower $\xi_{\mathrm{RH}}$ requires lower $g_{X}$ to obtain the correct relic density. This is because, at a given observable temperature $T$, colder hidden sectors imply lower equilibrium number densities. To keep the relic density constant, $g_{X}$ must be lower so that freezeout happens earlier. This is consistent with the results shown in Fig. 4 .

Finally, note that the $\xi_{\mathrm{RH}}=0.3$ contour ends at $m_{X} \simeq 45 \mathrm{keV}$. This is because the criterion that dark matter freezeout while non-relativistic, that is, $x_{f}^{h} \gtrsim 3$, is violated below this mass. In Fig. 8, we plot, as a function of $\xi_{\mathrm{RH}}$, the lower mass limit resulting from requiring that freezeout occurs with $x_{f}^{h} \gtrsim 3$ and yields $\Omega h^{2}=0.11$. We can see that the lower mass limit goes up with smaller $\xi_{\mathrm{RH}}$. This is because for colder hidden sectors, freezeout occurs earlier, and to satisfy $x_{f}^{h} \gtrsim 3$, we need larger $m_{X}$. This exercise establishes that the WIMPless dark matter framework may be valid at least down to dark matter masses of $m_{X} \sim \mathrm{keV}$. Below this mass, freezeout is not non-relativistic, and our analysis breaks down. It would be interesting to perform a more precise analysis without assuming non-relativistic freezeout, but this is beyond the scope of our study. 


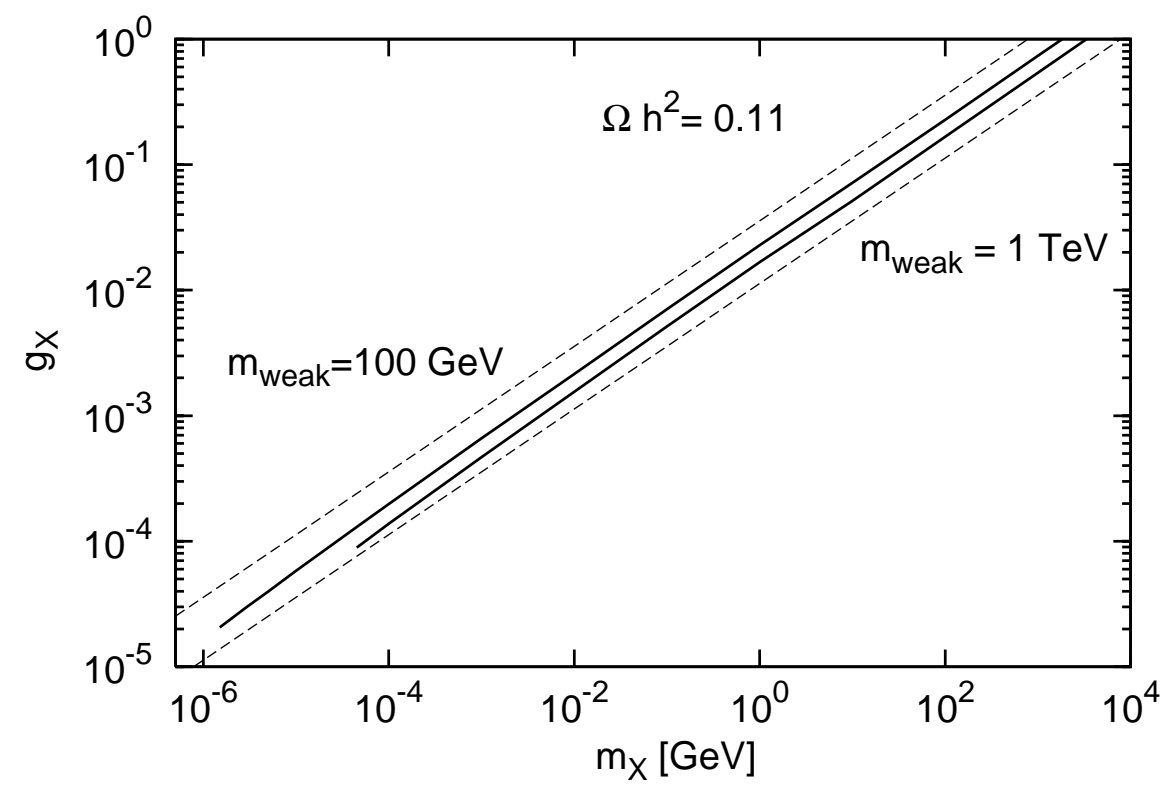

FIG. 7: Contours of $\Omega_{X} h^{2}=0.11$ in the $\left(m_{X}, g_{X}\right)$ plane for $\xi_{\mathrm{RH}}=0.8$ (upper solid) and 0.3 (lower solid), where the hidden sector is a 1-generation flavor-free version of the MSSM, corresponding to Model A (for $m_{X} \geq 1 \mathrm{MeV}$ ) or Model $\mathrm{C}$ (for $m_{X}<1 \mathrm{MeV}$ ). The minimum $m_{X}$ consistent with the requirement of non-relativistic freezeout depends strongly on $\xi_{\mathrm{RH}}$ (see text). Also plotted are lines of $m_{\text {weak }} \equiv\left(m_{X} / g_{X}^{2}\right) g^{\prime 2}=100 \mathrm{GeV}$ (upper dashed) and $1 \mathrm{TeV}$ (lower dashed).

\section{IMPLICATIONS OF CONNECTORS}

Throughout this work, we have ignored the possible existence of connector particles $Y$ with both SM and hidden gauge charges. If these are added, do they significantly affect the conclusions derived above?

There are two significant roles that connector particles can have. First, they likely establish thermal connectivity between the observable and hidden sectors when the temperatures are above the connector mass scale $m_{Y}$. At temperatures below $m_{Y}$, however, the hiddenSM interactions become weak, and the observable and hidden sectors decouple. As shown in Figs. 1] and 2, the BBN and CMB constraints actually allow, for example, a 1-generation flavor-free version of the MSSM with $m_{X} \lesssim T_{\mathrm{BBN}}^{h}$, even when $\xi_{\mathrm{RH}}=1$. In this case, then, there is no problem if the two sectors are in thermal contact right after reheating. For other hidden sectors, it may be possible that the existence of connectors enforces $\xi_{\mathrm{RH}}=1$, or alternatively, the assumption of $\xi_{\mathrm{RH}} \neq 1$ implies an upper bound on the reheating temperatures. One must check on a case by case basis whether connector particles upset the premises of our calculations.

A second affect is that connector particles open new avenues for dark matter annihilation. As an example, we assume that fermionic connector particles $Y$ couple through Yukawa couplings $\lambda X \bar{Y} f$, where $f$ is a SM fermion. We expect $m_{Y} \sim \max \left(m_{X}, m_{\text {weak }}\right)$ [30], and also require $m_{Y}>m_{X}$ to prevent the decay $X \rightarrow Y f$.

Such connector interactions induce annihilations $X X \rightarrow \bar{f} f$ through processes with $Y$ 


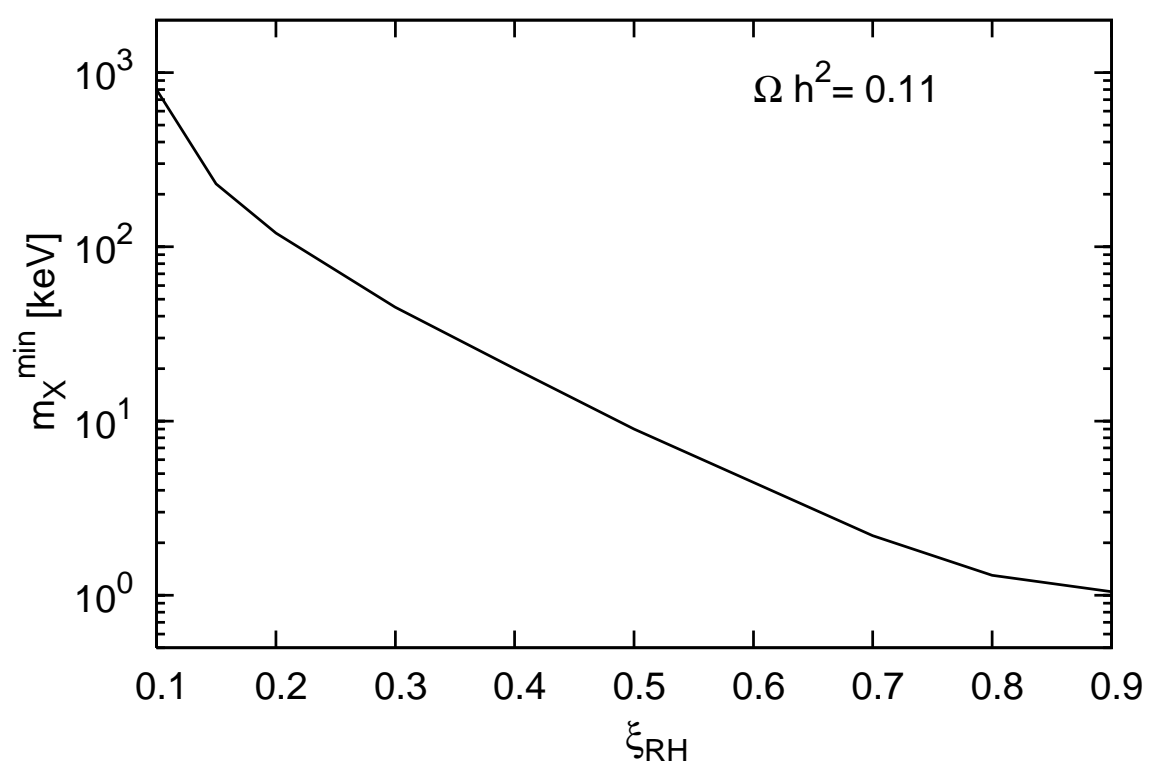

FIG. 8: Lower bound on the dark matter particle mass $m_{X}$ for different $\xi_{\mathrm{RH}}$ in the WIMPless scenario. As in Fig. 7, the hidden sector is a 1-generation flavor-free version of the MSSM, corresponding to Model A (for $m_{X} \geq 1 \mathrm{MeV}$ ) or Model C (for $m_{X}<1 \mathrm{MeV}$ ).

particles in the $t$-channel. There are two cases. If $m_{X} \lesssim m_{\text {weak }}, m_{Y} \sim m_{\text {weak }}$, and

$$
\langle\sigma v\rangle_{\bar{f} f} \sim \frac{\lambda^{4} m_{X}^{2}}{m_{Y}^{4}} \lesssim \frac{\lambda^{4}}{m_{Y}^{2}} \sim \frac{\lambda^{4}}{m_{\mathrm{weak}}^{2}} .
$$

On the other hand, if $m_{X} \gtrsim m_{\text {weak }}, m_{Y} \sim m_{X}$, and

$$
\langle\sigma v\rangle_{\bar{f} f} \sim \frac{\lambda^{4}}{m_{X}^{2}} \lesssim \frac{\lambda^{4}}{m_{\text {weak }}^{2}} .
$$

In either case, the annihilation cross section to SM particles is small relative to the completely hidden annihilation cross sections $g_{\text {weak }}^{4} / m_{\text {weak }}^{2}$, provided $\lambda \lesssim g_{\text {weak }} \simeq 0.65$. This is a rather weak criterion, and so typically, annihilation to SM particles is sub-dominant, and the analysis given above holds even in the presence of connector particles.

\section{CONCLUSIONS}

In the paper, we have studied the possibility that dark matter is hidden, that is, has no SM gauge interactions. Hidden sectors appear in many frameworks for physics beyond the SM, and hidden dark matter is perfectly viable, given all observations to date. We are particularly motivated by the recent proposal of WIMPless dark matter. As with WIMPs, WIMPless dark matter has the key virtue that its thermal relic density is in the right range to be cold dark matter. In contrast to WIMPs, however, WIMPless dark matter may have masses and couplings that differ drastically from WIMPs.

Although hidden sectors interact very weakly with the SM, hidden particles contribute to the energy density of the Universe. This impacts the expansion rate of the Universe, and 
so bounds from BBN and the CMB constrain hidden sectors. As is well known, current constraints completely exclude a hidden sector that is an exact copy of the MSSM. We find, however, that these constraints are rather brittle and easily avoided if the hidden sector is just slightly colder than the observable sector or if the mass scale of hidden superpartners differs from the MSSM. Several viable examples are discussed in Sec. II, and Figs. 1 and 2 give model-independent bounds that can be used to determine the viability of other models.

In Sec. III, we then defined a concrete model, a 1-generation flavor-free version of the MSSM. This model has features generic to GMSB models and provides a WIMPless dark matter candidate, the hidden stau $\tilde{\tau}_{R}^{h}$. The $\tilde{\tau}_{R}^{h}$ relic density depends sensitively on only a small number of parameters. This relic density was examined in Sec. IV by solving the Boltzmann equation numerically, and understanding these results through well-known analytic approximations, generalized to hidden sectors with different temperatures. These results confirm that in WIMPless models with $m_{X} \propto g_{X}^{2}$, the relic density is naturally in the right range for dark matter masses $\mathrm{keV} \lesssim m_{X} \lesssim \mathrm{TeV}$, greatly extending the conventional WIMP mass range. WIMPless dark matter therefore provides a class of dark matter candidates that shares the key relic density virtue of WIMPs. This generalization may have interesting applications, given the diverse and interesting phenomenology and observational anomalies already present at the $\mathrm{keV}, \mathrm{MeV}, \mathrm{GeV}$, and $\mathrm{TeV}$ mass scales.

We have determined the allowed range of $m_{X}$ by requiring that this dark matter freezes out with the right relic density while non-relativistic. Of course, this range may be further constrained by other considerations. In particular, the lightest WIMPless candidates, with $m_{X} \sim \mathrm{keV}$, may have interesting implications for structure formation. After freeze out, they can still couple to the hidden sector thermal bath through elastic scattering processes $\tilde{\tau}_{R}^{h} \gamma^{h} \rightarrow \tilde{\tau}_{R}^{h} \gamma^{h}$ and $\tilde{\tau}_{R}^{h} \nu^{h} \rightarrow \tilde{\tau}_{R}^{h} \nu^{h}$. After their kinetic decoupling at temperature $T_{\mathrm{kd}}$, they then stream freely between over- and under-dense regions. Free-streaming of thermal relics causes damping in density perturbations [52] at a comoving scale $\lambda_{\mathrm{FS}} \propto m_{X}^{-1 / 2} T_{\mathrm{kd}}^{-1 / 2}$; in contrast to WIMPs [53, 54], this is the dominant effect for $\mathrm{MeV}$ or lighter dark matter [55]. If this scale falls in the range $(1-80) h^{-1} \mathrm{Mpc}$, the linear matter power spectrum from high-resolution Lyman- $\alpha$ forest data can be used to place a lower bound on the mass of the dark matter particle. Current lower mass limits on warm dark matter of $0.55-\mathcal{O}(1) \mathrm{keV}$ from Lyman- $\alpha$ data [56, 57, 58, 59] therefore imply lower mass bounds in the WIMPless scenario, which depend on the kinetic decoupling temperature $T_{\mathrm{kd}}$ for different $\xi_{\mathrm{RH}}$.

In addition, another kind of dark matter lower mass limit follows from the observation that the phase-space density of the dark matter particles in galaxy halos cannot exceed the maximum value of the phase-space density when the dark matter particles were in kinetic equilibrium [60]. With knowledge of the WIMPless dark matter's chemical potential, one can then apply these generalized Tremaine-Gunn bounds from Ref. [61] to our scenario. Detailed considerations of these effects are beyond the scope of this study, but they may modify the lower bound on $m_{X}$ or, alternatively, provide interesting astrophysical signals for the hidden dark matter scenarios discussed here.

\section{Acknowledgments}

We thank Manoj Kaplinghat and Jason Kumar for many helpful discussions. JLF and HT are supported in part by NSF grants PHY-0239817, PHY-0314712, and PHY-0653656, NASA grant NNG05GG44G, and the Alfred P. Sloan Foundation. HY is supported by NSF 
grant PHY-0709742.

[1] S. Dimopoulos, D. Eichler, R. Esmailzadeh and G. D. Starkman, Phys. Rev. D 41, 2388 (1990).

[2] G. D. Starkman, A. Gould, R. Esmailzadeh and S. Dimopoulos, Phys. Rev. D 41, 3594 (1990).

[3] T. D. Lee and C. N. Yang, Phys. Rev. 104, 254 (1956).

[4] S. I. Blinnikov and M. Y. Khlopov, Sov. J. Nucl. Phys. 36, 472 (1982) [Yad. Fiz. 36, 809 (1982)]; Sov. Astron. 27, 371 (1983) [Astron. Zh. 60, 632 (1983)].

[5] H. M. Hodges, Phys. Rev. D 47, 456 (1993).

[6] Z. G. Berezhiani, A. D. Dolgov and R. N. Mohapatra, Phys. Lett. B 375, 26 (1996) arXiv:hep-ph/9511221.

[7] R. N. Mohapatra and V. L. Teplitz, Phys. Rev. D 62, 063506 (2000) arXiv:astro-ph/0001362.

[8] Z. Berezhiani, D. Comelli and F. L. Villante, Phys. Lett. B 503, 362 (2001) arXiv:hep-ph/0008105.

[9] R. N. Mohapatra, S. Nussinov and V. L. Teplitz, Phys. Rev. D 66, 063002 (2002) arXiv:hep-ph/0111381.

[10] A. Y. Ignatiev and R. R. Volkas, Phys. Rev. D 68, 023518 (2003) arXiv:hep-ph/0304260.

[11] R. Foot, Phys. Rev. D 69, 036001 (2004) arXiv:hep-ph/0308254].

[12] Z. Berezhiani, P. Ciarcelluti, D. Comelli and F. L. Villante, Int. J. Mod. Phys. D 14, 107 (2005) arXiv:astro-ph/0312605].

[13] R. Foot and R. R. Volkas, Phys. Rev. D 70, 123508 (2004) arXiv:astro-ph/0407522.

[14] M. B. Green and J. H. Schwarz, Phys. Lett. B 149, 117 (1984).

[15] D. J. Gross, J. A. Harvey, E. J. Martinec and R. Rohm, Phys. Rev. Lett. 54, 502 (1985).

[16] R. Blumenhagen, M. Cvetic, P. Langacker and G. Shiu, Ann. Rev. Nucl. Part. Sci. 55, 71 (2005) arXiv:hep-th/0502005.

[17] R. Schabinger and J. D. Wells, Phys. Rev. D 72, 093007 (2005) arXiv:hep-ph/0509209.

[18] B. Patt and F. Wilczek, arXiv:hep-ph/0605188.

[19] M. J. Strassler and K. M. Zurek, Phys. Lett. B 651, 374 (2007) arXiv:hep-ph/0604261.

[20] H. Georgi, Phys. Rev. Lett. 98, 221601 (2007) arXiv:hep-ph/0703260.

[21] J. Kang and M. A. Luty, arXiv:0805.4642 [hep-ph].

[22] X. Chen and S. H. Tye, JCAP 0606, 011 (2006) arXiv:hep-th/0602136].

[23] T. Kikuchi and N. Okada, Phys. Lett. B 665, 186 (2008) [arXiv:0711.1506 [hep-ph]].

[24] J. March-Russell, S. M. West, D. Cumberbatch and D. Hooper, JHEP 0807, 058 (2008) arXiv:0801.3440 [hep-ph]].

[25] D. Hooper and K. M. Zurek, Phys. Rev. D 77, 087302 (2008) [arXiv:0801.3686 [hep-ph]].

[26] J. McDonald and N. Sahu, JCAP 0806, 026 (2008) arXiv:0802.3847 [hep-ph]].

[27] Y. G. Kim, K. Y. Lee and S. Shin, JHEP 0805, 100 (2008) arXiv:0803.2932 [hep-ph]].

[28] W. Krolikowski, arXiv:0803.2977 [hep-ph].

[29] Y. Gong and X. Chen, arXiv:0803.3223 [astro-ph].

[30] J. L. Feng and J. Kumar, arXiv:0803.4196 [hep-ph].

[31] R. Foot, arXiv:0804.4518 [hep-ph].

[32] J. L. Feng, J. Kumar and L. E. Strigari, arXiv:0806.3746 [hep-ph].

[33] See, e.g., E. W. Kolb, D. Seckel and M. S. Turner, Nature 314, 415 (1985).

[34] R. Foot and R. R. Volkas, Astropart. Phys. 7, 283 (1997) arXiv:hep-ph/9612245. 
[35] G. Steigman, D. N. Schramm and J. E. Gunn, Phys. Lett. B 66 (1977) 202.

[36] For a recent review, see G. Steigman, Ann. Rev. Nucl. Part. Sci. 57 (2007) 463 arXiv:0712.1100 [astro-ph]].

[37] R. H. Cyburt, B. D. Fields, K. A. Olive and E. Skillman, Astropart. Phys. 23 (2005) 313 arXiv:astro-ph/0408033].

[38] B. Fields and S. Sarkar, arXiv:astro-ph/0601514.

[39] E. Komatsu et al. [WMAP Collaboration], arXiv:0803.0547 [astro-ph].

[40] [Planck Collaboration], arXiv:astro-ph/0604069.

[41] S. Hannestad, H. Tu and Y. Y. Y. Wong, JCAP 0606 (2006) 025 arXiv:astro-ph/0603019.

[42] J. Hamann, S. Hannestad, G. G. Raffelt and Y. Y. Y. Wong, JCAP 0708 (2007) 021 arXiv:0705.0440 [astro-ph]].

[43] K. Ichikawa, T. Sekiguchi and T. Takahashi, arXiv:0803.0889 [astro-ph].

[44] L. A. Popa and A. Vasile, JCAP 0806 (2008) 028 [arXiv:0804.2971 [astro-ph]].

[45] V. Simha and G. Steigman, JCAP 0806 (2008) 016 [arXiv:0803.3465 [astro-ph]].

[46] G. Mangano, G. Miele, S. Pastor, T. Pinto, O. Pisanti and P. D. Serpico, Nucl. Phys. B 729 (2005) 221 arXiv:hep-ph/0506164.

[47] T. S. Coleman and M. Roos, Phys. Rev. D 68 (2003) 027702 arXiv:astro-ph/0304281].

[48] A. Pukhov et al., arXiv:hep-ph/9908288.

[49] A. Pukhov, arXiv:hep-ph/0412191.

[50] P. Gondolo and G. Gelmini, Nucl. Phys. B 360 (1991) 145.

[51] P. Gondolo, J. Edsjo, P. Ullio, L. Bergstrom, M. Schelke and E. A. Baltz, JCAP 0407 (2004) 008 arXiv:astro-ph/0406204.

[52] E. Bertschinger, Phys. Rev. D 74 (2006) 063509 arXiv:astro-ph/0607319].

[53] A. Loeb and M. Zaldarriaga, Phys. Rev. D 71, 103520 (2005) arXiv:astro-ph/0504112].

[54] S. Profumo, K. Sigurdson and M. Kamionkowski, Phys. Rev. Lett. 97, 031301 (2006) arXiv:astro-ph/0603373.

[55] D. Hooper, M. Kaplinghat, L. E. Strigari and K. M. Zurek, Phys. Rev. D 76 (2007) 103515 arXiv:0704.2558 [astro-ph]].

[56] V. K. Narayanan, D. N. Spergel, R. Dave and C. P. Ma, Astrophys. J. 543 (2000) L103 arXiv:astro-ph/0005095].

[57] M. Viel, J. Lesgourgues, M. G. Haehnelt, S. Matarrese and A. Riotto, Phys. Rev. D 71 (2005) 063534 arXiv:astro-ph/0501562.

[58] K. Abazajian, Phys. Rev. D 73 (2006) 063513 [arXiv:astro-ph/0512631.

[59] M. Viel, G. D. Becker, J. S. Bolton, M. G. Haehnelt, M. Rauch and W. L. W. Sargent, Phys. Rev. Lett. 100 (2008) 041304 arXiv:0709.0131 [astro-ph]].

[60] S. Tremaine and J. E. Gunn, Phys. Rev. Lett. 42 (1979) 407.

[61] J. Madsen, Phys. Rev. D 44 (1991) 999. 